\title{
Clear Cell Myoepithelial Carcinoma of the Upper Lip - A Rare Case Report
}

\author{
Swati GOTMARE ${ }^{1}$, Anish GUPTA ${ }^{2}$, Megha JAIN², Mandavi WAGHMARE ${ }^{3}$ \\ Department of Oral Pathology, 'DY Patil University School of Dentistry, MUMBAI, INDIA, ${ }^{2}$ People's Dental Academy, BHOPAL, INDIA \\ ${ }^{3}$ Department of Oral Medicine \& Radiology, DY Patil University School of Dentistry, MUMBAI, INDIA
}

\begin{abstract}
Myoepithelial carcinomas are quite infrequent neoplasms and coupled with their diverse morphological appearance are interesting as far as diagnosis and management is concerned. They account for less than $1 \%$ of all salivary gland tumors. The variable morphologic appearance of myoepithelial carcinoma leads to a wide differential diagnosis, including primary salivary gland tumors and metastatic tumors. The prognosis of these tumors is not fair as they are locally aggressive and approximately one third of the patients die of the pathology. We report a case of clear cell variant of myoepithelial carcinoma in an unusual location, i.e. the upper lip. The treatment carried out was wide surgical resection. The patient was followed up for 2 years and was symptom free. The clear cell variant of myoepithelial carcinoma is extremely rare and only about 51 cases of this variant affecting the salivary glands have been reported worldwide so far.
\end{abstract}

Key Words: Myoepithelial carcinoma, Clear cell carcinoma, Upper lip

\section{INTRODUCTION}

Salivary gland neoplasms pose great challenges in management because of their varied morphology and unpredictable behavior. Myoepithelial Carcinomas (MC) account for 0.4 to $0.6 \%$ of all salivary gland neoplasms (1). This neoplasm was introduced by Stromeyer et al. in 1975 . Parotid glands are affected most commonly, followed by the submandibular and minor salivary glands. The most common site for intra-oral presentation is the palate (2). There is usually a precursor lesion such as pleomorphic adenoma, myoepithelioma associated with it but it may also arise de novo (3). MC is difficult to diagnose clinically and histopathologically and may mimic a benign neoplasm. Immunohistochemical (IHC) markers such as CAM5.2, $\mathrm{AE} 1 \mathrm{AE} 3,34 \beta \mathrm{E} 12$, cytokeratin 7 , cytokeratin 14 , vimentin, S-100 protein, smooth muscle actin, CD 10, calponin, muscle-specific actin, glial fibrillary acidic protein, carcinoembryonic antigen, and epithelial membrane antigen have been used to confirm the diagnosis $(4,5)$. We report a rare case of Clear Cell Myoepithelial Carcinoma (CCMC) of the upper lip. To the best of our knowledge, this is the first case to be reported in the upper lip and histopathologically presented as a clear cell variant. A panel of IHC markers was applied to confirm the diagnosis.

\section{CASE REPORT}

A 46-year-old female patient reported to us with a complaint of mild extra-oral and intra-oral swelling for

(Turk Patoloji Derg 2019, 35:79-82)

Received : 19.05.2016 Accepted : 07.10.2016 the last 6 months. Extra-orally the swelling was extending superior-inferiorly from the base of the nose to the philtrum and medio-laterally from the midline to the centre of the upper lip on the left side with an approximate size of $3 \times 2 \mathrm{~cm}$. Intra-orally, a diffuse swelling obliterating the labial vestibule extending from the maxillary right central incisor and crossing the midline up to the left canine region was seen. The swelling was soft to firm on palpation with a smooth glistening surface appearing similar to the surrounding mucosa (Figure 1). Occlusal radiograph showed involvement of the medial and lateral alveolar process of maxillary left central incisor with horizontal bone loss and saucerization. CT scan revealed an expansile lesion involving the maxillary anterior region causing expansion of the buccal cortical plate (Figure 2).

The patient's personal and family history were noncontributory. Metastasis from primary tumor of other sites was ruled out. There was no evidence of any metastasis. Blood investigations were within normal limits. A provisional diagnosis of salivary gland tumor was made and accordingly an incisional biopsy was performed which revealed malignancy of salivary gland. The tumor was surgically removed and submitted for histopathological examination. Gross specimen measured around $3 \times 2$ $\mathrm{cm}$, was non-encapsulated, multinodular and presented irregular borders. It was firm in consistency and grayish white in color. Microscopy showed tumor nodules composed of epitheloid cells arranged in diffuse sheets,

Correspondence: Anish GUPTA

People's Dental Academy, Department of Oral Pathology, BHOPAL, INDIA

E-mail: anishpooja687@yahoo.co.in Phone: +88 17173332 
nests and cords separated by fibrous septa. Tumor cells had round to oval nuclei, vesicular chromatin, prominent nucleoli and a moderate amount of eosinophilic cytoplasm. Mitotic figures ranged from 5 to 7 per 10 high power field. Many of the cells showed partially clear cytoplasm (Figure 3). The stroma contained hyalinised material. No areas of necrosis were evident. There was presence of invasion in the adjacent alveolar bone. A panel of IHC markers consisting of alpha-SMA (Figure 4), HMW cytokeratin (Figure 5), calponin (Figure 6), CD-10 (Figure 7) and EMA were applied. They were all indicated by dark brown cytoplasmic staining. The results were positive for all the markers, except EMA. The diagnosis was confirmed as CCMC. The patient was followed up for 2 years and there was no complaint or recurrence.

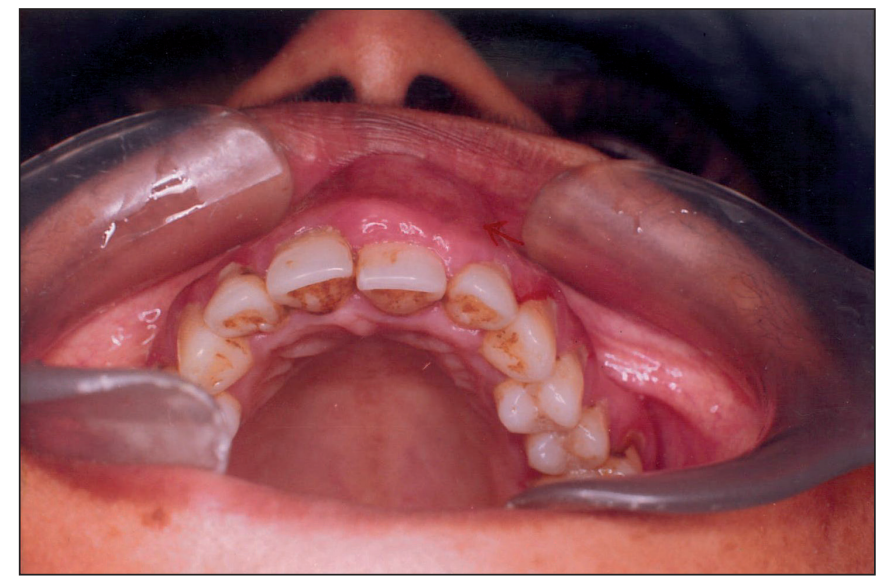

Figure 1: Intra-oral presentation reveals obliteration of labial vestibule.

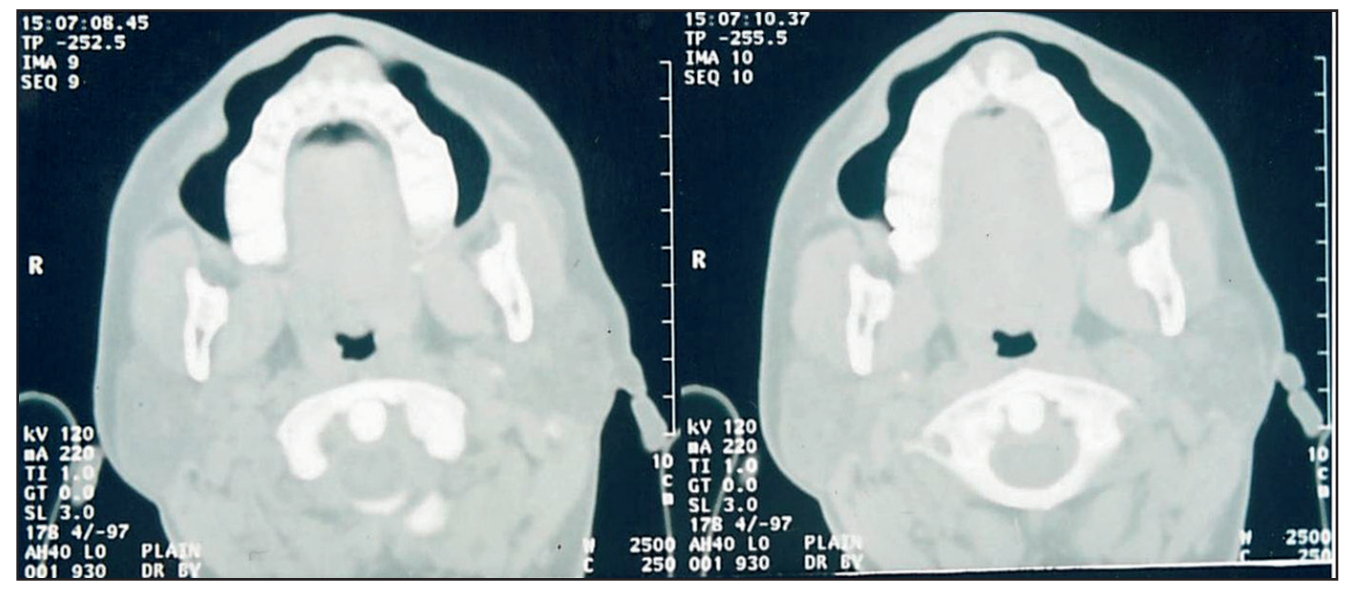

Figure 2: CT scan reveals an expansile swelling.

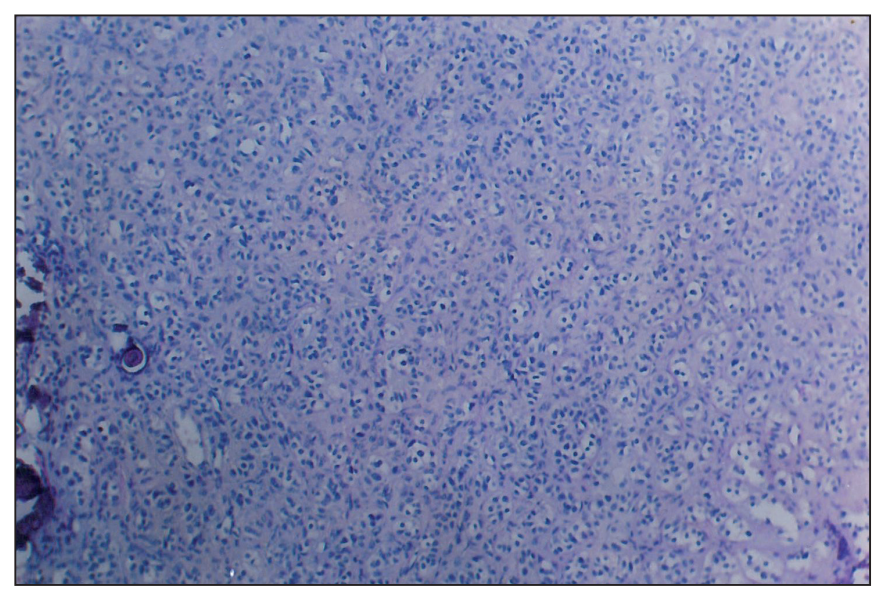

Figure 3: Neoplasm showing mixture of epitheloid and clear cells (devoid of cytoplasm) in a hyalinised stroma and cytological pleomorphism (H\&E; 100).

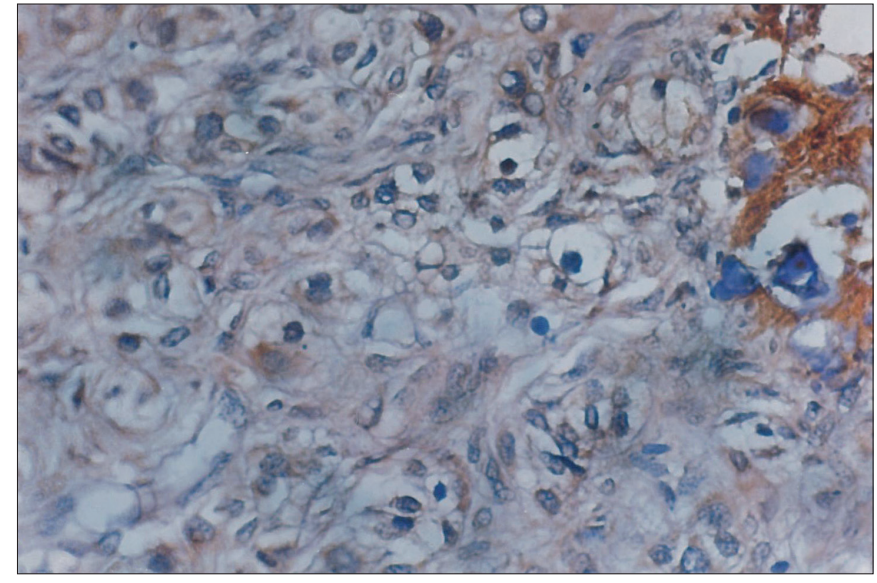

Figure 4: Tumor cells are positive for alpha- SMA (SMA; x400). 


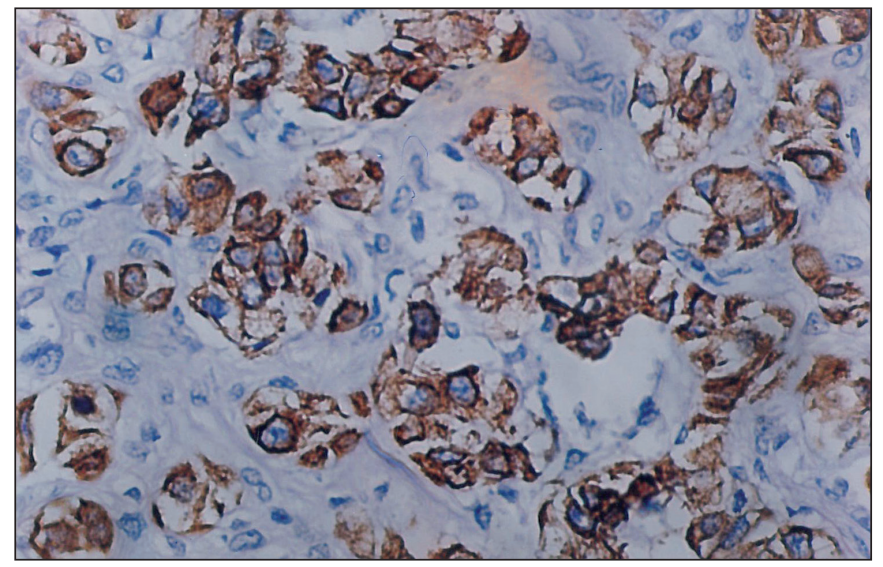

Figure 5: Tumor cells are positive for high molecular weight cytokeratin (HMWCK; x400).

\section{DISCUSSION}

Myoepithelial cells were first introduced by Krause in 1865 in breast tissue followed by its observation in salivary gland acini (6). The physiologic roles of these cells are contraction, propagation of neural stimuli, basement membrane production, transport of metabolites and tumour suppression (6,7). MC or malignant myoepithelioma is defined as a malignant epithelial neoplasm in which the predominant differentiation of the tumor cells is myoepithelial (8). There is equal sex distribution in the various reported cases. It is believed that a precursor lesion such as pleomorphic adenoma may be present or MC may arise de novo. Some authors believe that de novo MCs are more aggressive and may have a poor prognosis (9). The final outcome of MC is controversial in either type of case (5). Major salivary glands are more commonly affected than the minor ones although a few studies have reported conflicting results $(2,4,3)$. The CCMC has been reported in the parotid gland, submandibular gland and intra-orally in the palate, retromolar area, maxillary sinus and even the base of the tongue. To the best of our knowledge, this variant in the unusual location of the upper lip is the first to be reported. The presentation of neoplastic myoepithelial cells in MC is the major cause of a diagnostic dilemma as they occur in epitheloid (most predominant), spindle, plasmacytoid and clear forms (8). The tumor, along with the cells, also shows myxoid or hyaline-like material in the stroma (10). In our case, there was mixture of epitheloid and clear cells (percentage of clear cells being more than $50 \%$ ) and the matrix showed hyaline material. MCs may resemble a benign tumor and the features demarcating MCs from benign tumors are cytological pleomorphism, mitosis, necrosis and invasion of adjacent tissues. Khademi B et al. have shown that preoperative diagnosis

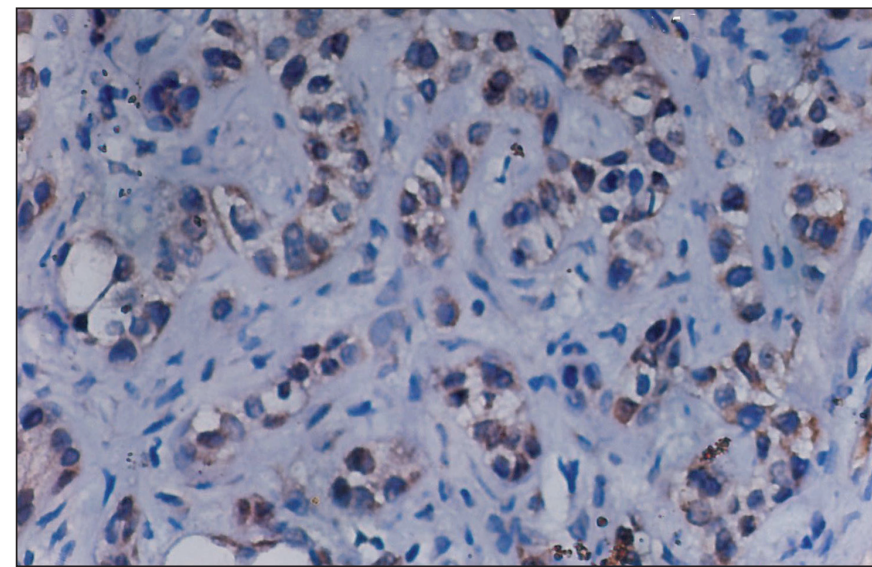

Figure 6: Tumor cells are positive for calponin (Calponin; $\mathrm{x} 400$ ).

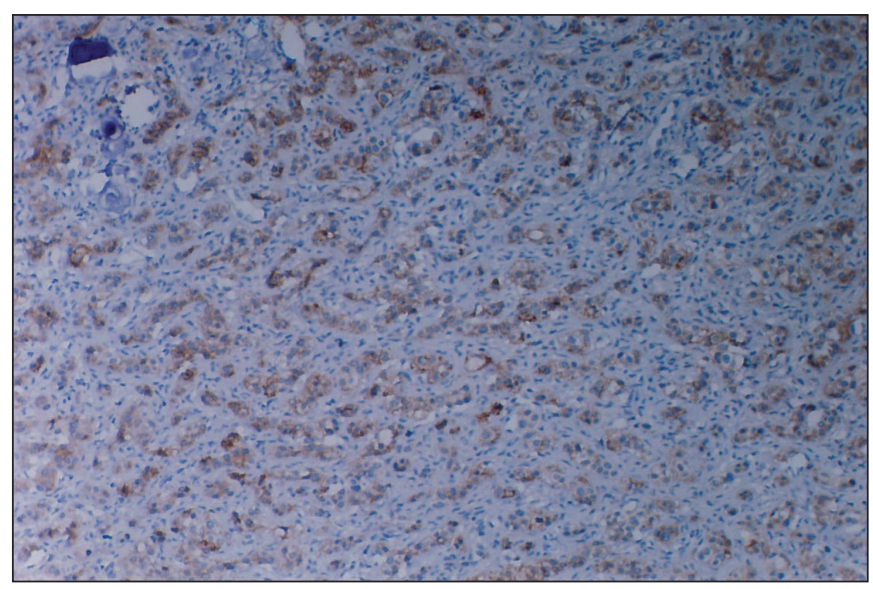

Figure 7: Tumor cells are positive for CD10 (CD10; x100).

of malignant myoepitheliomas is unreliable (11). The nuclei show cytological atypical changes in the form of pleomorphism, mitotic activity, and vesicular chromatin. $\mathrm{MC}$ of spindle cell type is rare, having a clinical presentation as an ulcerative lesion, and has a poor prognosis (12). The clear cells of CCMC display typical signet ring or lipoblast shapes, although there may be admixture of other cells such as epitheloid, spindle or plasmacytoid $(13,14)$. It has been reported that MC may show presence of osteoclast-like giant cells and the prognosis is adversely affected when they are present (5). Myoepithelial cells are capable of producing basement membrane and nonbasement membrane proteins (chiefly chondroitin sulfate proteoglycans), which leads to formation of a myxoid stroma (15). IHC markers such as S-100, alpha-SMA, cytokeratins, calponin, EMA, and vimentin have been used to confirm the myoepithelial cells $(2,16)$. The cells show intense staining for these markers, although at times the staining may be sparse or negative. This may be attributed 
to the fact that the myoepithelial cells may show different stages of differentiation. It has been suggested that p53 can be a useful predictor for prognosis. Its over-expression is directly related to compromised survival rates. CCMCs may harbor the Ewing sarcoma breakpoint region 1 (EWSR1) rearrangement and therefore may be genetically related to EWSR1-rearranged cutaneous myoepithelial tumors (17). The features of our case were consistent with the criteria of CCMC. Due to scanty literature on CCMC and moreover the rare location of the upper lip, further longitudinal studies may be required. The treatment for this tumor is wide surgical resection and a close follow-up. The prognosis of CCMC is not very favourable.

In conclusion, MCs are extremely noteworthy tumors that mostly affect major salivary glands. Very few cases have been documented in the oral cavity. The tumor cells may presented as epitheloid, spindle or plasmacytoid types. The clear cell variant is extremely rare and we report this variant in a very unusual location, the upper lip, invading the alveolar bone. The prognosis of this tumor is guarded. The only treatment is surgical resection with wide margins.

\section{REFERENCES}

1. Jones AV, Craig GT, Speight PM, Franklin CD. The range and demographics of salivary gland tumors diagnosed in a UK population. Oral Oncol.2008;44:407-17.

2. Lata J, Ahmad F, Chand V. Myoepithelial carcinoma of buccal mucosa: A rare tumor. Contemp Clin Dent.2014;5:247-51.

3. Kane SV, Bagwan IN. Myoepithelial carcinoma of the salivary glands: A clinicopathologic study of 51 cases in a tertiary cancer center. Arch Otolaryngol Head Neck Surg. 2010;136:702-12.

4. Savera AT, Sloman A, Huvos AG, Klimstra DS. Myoepithelial carcinoma of the salivary glands: A clinicopathologic study of 25 patients. Am J Surg Pathol. 2000;24:761-74.

5. Nagao T, Sugano I, Ishida Y, Tajima Y, Matsuzaki O, Konno A, Kondo Y, Nagao K. Salivary gland malignant myoepithelioma: A clinicopathologic and immunohistochemical study of ten cases. Cancer. 1998;83:1292-9.
6. Chitturi RT, Veeravarmal V, Nirmal RM, Reddy BV. Myoepithelial Cells (MEC) of the salivary glands in health and tumours. J Clin Diagn Res. 2015;9:ZE14-8.

7. Redman RS. Myoepithelium of salivary glands. Microsc Res Tech. 1994;27:25-45.

8. Simpson RHW. Myoepithelial tumours of the salivary glands. Curr Diagn Pathol. 2002;8:328-37.

9. Di Palma S, Guzzo M. Malignant myoepithelioma of salivary glands: Clinicopathological features of ten cases. Virchows Arch A Pathol Anat Histopathol. 1993;423:389-96.

10. Yu G, Qu G, Kong L, Pan X, Wang W, Lv J. Primary myoepithelial carcinoma of the larynx: Case report and review of the literature. Pathol Res Pract. 2011;207:127-30.

11. Khademi B, Kazemi T, Bayat A, Bahranifard H, Daneshbod Y, Mohammadianpanah M. Salivary gland myoepithelial neoplasms: A clinical and cytopathologic study of 15 cases and review of the literature. Acta Cytol. 2010;54:1111-7.

12. Kane SV, Bagwan IN, Shet T, Bavi P, D’Cruz A. Spindle cell myoepithelial carcinoma of the oral cavity-A report of two cases. Oral Oncol Extra. 2006;42:66-9.

13. Park JH, Choi JY, Hong R, DO NY. Clear cell myoepithelial carcinoma in the base of the tongue: Case report and review of the literature. Oncol Lett. 2012;4:1241-3.

14. Michal M, Skálová A, Simpson RH, Rychterová V, Leivo I. Clear cell malignant myoepithelioma of the salivary glands. Histopathology. 1996;28:309-15.

15. Ingle $\mathrm{Y}$, Shah AA, Kheur S, Routaray S. Myoepithelial cell carcinoma of the oral cavity: A case report and review of literature. J Oral Maxillofac Pathol. 2014;18:472-7.

16. Losito NS, Botti G, Ionna F, Pasquinelli G, Minenna P, Bisceglia M. Clear-cell myoepithelial carcinoma of the salivary glands: A clinicopathologic, immunohistochemical, and ultrastructural study of two cases involving the submandibular gland with review of the literature. Pathol Res Pract. 2008;204:335-44.

17. Skálová A, Weinreb I, Hyrcza M, Simpson RH, Laco J, Agaimy A, Vazmitel M, Majewska H, Vanecek T, Talarčik P, Manajlovic S, Losito SN, Šteiner P, Klimkova A, Michal M. Clear cell myoepithelial carcinoma of salivary glands showing EWSR1 rearrangement: Molecular analysis of 94 salivary gland carcinomas with prominent clear cell component. Am J Surg Pathol. 2015;39:338-48. 